\title{
On the proper domain of psychological predicates
}

\author{
Carrie Figdor
}

Received: 7 April 2014 / Accepted: 5 November 2014

(C) Springer Science+Business Media Dordrecht 2014

\begin{abstract}
One question of the bounds of cognition is that of which things have it. A scientifically relevant debate on this question must explain the persistent and selective use of psychological predicates to report findings throughout biology: for example, that neurons prefer, plants and fruit flies decide, and bacteria communicate linguistically. This paper argues that these claims should enjoy default literal interpretation, and that these reports of psychological properties in non-humans are as straightforward as they seem. An epistemic consequence is that these findings can contribute directly to understanding the nature of psychological capacities.
\end{abstract}

Keywords Cognition - Psychological predicates · Folk psychology · Verbs · Psychological explanation · Cognitive mechanisms $\cdot$ Metaphor

[A]ny mechanisms you can think of you will find-even if it is the most bizarre form of thinking. Anything. So if the material tells you 'it may be this', allow that. Don't turn it aside and call it an exception, an aberration.

Barbara McClintock (in Trewavas 2007, from Keller 1983)

\section{Two approaches to the metaphysics of psychology}

One question of the bounds of cognition is that of which things have it. ${ }^{1}$ Many now accept that non-human primates and at least some other animals have at least some cognitive capacities once thought exclusive to humans (e.g., Bekoff et al. 2002; Andrews

\footnotetext{
1 A popular, but distinct, question is whether cognitive processes are bound to-realized by-what's inside the skull or else extend through the body or into the environment. This boundary dispute arises downstream from the issue discussed here.
}

C. Figdor $(\varangle)$

Department of Philosophy, University of Iowa, 260 EPB, Iowa City, IA 52242, USA

e-mail: carrie-figdor@uiowa.edu 
2009). But do neurons prefer (Izhikevich 2007)? Do bacteria communicate linguistically (Ben-Jacob et al. 2012)? Do plants and fruit flies decide (Brenner et al. 2006; DasGupta et al. 2014)? What is remarkable about these and similar uses throughout the biological sciences is that they appear in peer-reviewed reports and reviews of experimental results that are in every other respect just like those accepted as straightforward accounts of new discoveries anywhere else:

(1) Four-month-olds prefer looking at motion displays that resemble human walking compared with displays simulating random motion (Bertenthal 1993).

(2) A resonator neuron prefers inputs having frequencies that resonate with the frequency of its subthreshold oscillations (Izhikevich 2007).

(3) Infants habituated to two jumps of a puppet dishabituated when shown three jumps, and vice versa (Wagner and Carey 2003; Wynn 1996).

(4) The orbitofrontal cortex neurons described here habituate more slowly than is typical of neurons with responses to novel visual stimuli recorded in the amygdala (Rolls et al. 2005).

These uses are consistent and may be integrated in the relevant disciplines for decades or more by leading scientists (including Darwin, as Brenner et al. op.cit., p. 415, point out):

(5) In preferring a slit specific in width and orientation this cell [with a complex receptive field] resembled certain cells with simple fields (Hubel and Wiesel 1962, p. 115).

(6) It is the response properties of the last class of units [of cells recorded via electrodes implanted in a rat's dorsal hippocampus] which has led us to postulate that the rat's hippocampus functions as a spatial map. ... These 8 units then appear to have preferred spatial orientations (O'Keefe and Dostrovsky 1971, p. 172).

A scientifically relevant debate on the bounds of cognition must explain these ascriptions, or explain them away.

This paper argues that these reports are as straightforward as they seem, because the capacities denoted by these and other common psychological verbs are the same across these disparate domains. ${ }^{2}$ This view, which I call Anti-Exceptionalism, sees biology as progressing in usual (if philosophically unexpected) ways, not regressing in timeworn ways. Its answer to Wimsatt's (2006) query-

\footnotetext{
2 The psychological predicates or verbs referred to throughout this paper comprise the vocabulary of common-sense or folk psychology (extensions in cognitive psychology are not critical here; in Sect. 2, I discuss psychological verb classes in linguistics.) Terms in this vocabulary are subject to revision, extension, precisification, or becoming irrelevant as the sciences proceed, but (as argued in the remainder of this paper) their grounding in our lay understanding of psychology is inclusive: their folk meanings easily adjust to discoveries about the capacities they pick out in the way that the folk physical term "gold" was adjusted to fit a deeper understanding of gold eventually provided by physics and chemistry. (I thank an anonymous reviewer for pressing me to clarify this point.)
} 
Memory-a property of molecules, neural circuits, tracts, hemispheres, brains-invats, embodied socialized enculturated beings, or institutions?

- is, in each domain, an empirically defeasible "yes", and ditto for other psychological capacities ascribed in them. From this perspective, the idea that top-notch scientists doing top-notch science are succumbing to a chronic human weakness for anthropomorphizing reflects a chronic human refusal to acknowledge how much we have in common with other living things. ${ }^{3}$

However, while Anti-Exceptionalism is primarily about psychological capacities, the initial objections to it are invariably expressed in semantic or conceptual terms: that these uses of psychological predicates are nonsensical, metaphorical, or differ in reference-that is, are merely behavioristic or instrumental in some sense. ${ }^{4}$ Bennett and Hacker (2003) present the nonsense objection for the specific case of neuroscience (see also Slaney and Maraun 2005), while Dennett and Searle reject the nonsense objection in favor of the intentional stance and the metaphorical objection respectively (Bennett et al. 2007, pp. 88-89 and 112). ${ }^{5}$ The metaphysics comes in via a presupposed conviction that only human manifestations of these capacities (or very close approximations thereof) are real or genuine (or "fully fledged", as Dennett puts it in Bennett et al. op. cit., p. 87). This humans-only (or anthroponormative) conviction is manifested as a claim about literal reference.

This metaphysico-semantic debate is largely independent of and arises prior to the epistemological concerns that dominate debates about animal cognition, when it arises at all. The first bastion of resistance (or outrage) is not that the scientists' reports are false or unjustified, but that they're cognitively defective: the immediate response is "They don't mean it literally", not "They don't have sufficient reason". ${ }^{6}$ As a result, this debate concerns the reference of psychological verbs and concepts, rather than Morgan's Canon or evolution. Indeed in many of these cases the language police can't readily call up the usual epistemic reinforcements, as standard frameworks of

\footnotetext{
3 Although I focus on biological cases, Anti-Exceptionalism is open in principle to ascriptions to nonbiological (i.e. artificial) cases. For a non-anthroponormative, biology-based view of cognition, see Lyon (2006).

4 An indirect way of raising one or another these objections is to reserve the label "mental" for human cases of the psychological capacities and quarantine the rest in a separate and unequal "cognitive" category. Unless the dispute is merely verbal — for example, one might simply assert that all mental states are conscious states-the specific objection can be made explicit by asking what counts as mental and what merely cognitive.

5 Dennett's intentional stance may be considered a sui generis version of the behavioristic or instrumental objection; in any case, he combines this with homuncular explanation (e.g., in Bennett et al. op.cit., pp. 88-89) such that what is ascribed at subpersonal levels, even if the same words are used, is not the same as what is ascribed to persons.

6 Thus, an anonymous reviewer commented on an earlier draft that in cases "when we attribute genuine mental states to adult humans ... reporting a preference is an act that has an extraordinary number of inferential consequences. In the case of neurons, it's no more than a colorful redescription of the observed behavior." This comment presupposes that behaviorism or instrumentalism is not true of people (about which Anti-Exceptionalism is officially neutral) and asserts a question-begging interpretation of what the neuron sentences say (about which it is not).
} 
comparative psychology, naïve anthropomorphism, or common ancestry often don't readily apply (Andrews 2009, 2014; Keeley 2004; Sober 2005). ${ }^{7}$

The relation between this debate and the standard philosophical debate may be explained by considering two ways to ask about the nature of a psychological trait. Using the timeworn example of belief, we can ask: (a) What are the conditions or kinds of evidence in virtue of which we are justified in saying something has beliefs? or (b) When is an ascription of "believes" literal? In both cases the metaphysical question is approached obliquely, in the first case via epistemology, in the second via semantics. The standard debate focuses on the first question. It revolves around competing theories of belief and whether non-human animals satisfy the defended criteria. One might hold a traditional view of believing as using a mental representation in a particular way, and debate whether representations require language or concepts (e.g. Davidson 1975; Stich 1979) or not (e.g., Bermudez 2003); alternatively, one might give a dispositional theory of belief (e.g., Schwitzgebel 2012). Either way, the debate presupposes that the human case is the norm; the only issue is whether we have sufficient evidence that other animals can justifiably be said to satisfy an account of belief modeled on the human case.

The second question arises because ascriptions of psychological properties to neurons and plants, inter alia, undermine this presupposition. They force us to consider whether we already know or can infer from the human case what these properties must involve. Or rather, the metaphysical conviction mentioned above generates a refusal to consider this possibility, and this refusal is expressed by means of rejecting the truth-aptness of the ascriptions themselves. In short, the metaphysical conviction is the metaphysical ground of the traditional debate, and the debate over literal use is about this ground. The epistemic consequences are also significant. If these ascriptions are literal, we will regard the discoveries they are used to describe as potential contributions to our understanding of psychological properties. If the ascriptions are cognitively defective, they have no such potential, and the traditional debate's anthroponormativity remains intact.

The objectors owe a defense of the non-literal interpretations entailed by their metaphysical conviction. On her part, the Anti-Exceptionalist must show how default literal interpretations make sense. ${ }^{8}$ I aim to discharge a key part of the Anti-Exceptionalist's burden in this paper. I largely ignore (here) the negative task of showing the implausibility of non-literal interpretation in order to focus on the positive task of showing

\footnotetext{
7 This is not to say that epistemic issues are not raised: for example, Alpi et al. (2007) question the explanatory utility of the concept of plant neurobiology; Trewavas agrees that this is a metaphor (though Alpi et al. call it an analogy) but defends its utility. (See also Calvo and Keijzer 2009 and Brenner et al. 2007).

8 This is not to affirm, with panpsychism, that they are literal everywhere. My perspective has a contemporary parallel in ojalehto et al. (2013), who defend teleological concepts in science; and my strategy has an historic precedent in Fellbaum and Miller's (1990) reply to Rips and Conrad's (1989) claim that certain patterns of mental verb use characterize folk beliefs about mental activities. They reply that verbal and nominal semantic relations are not the same and that psychological verbs follow the patterns for verbs in general. Rips and Conrad (1990) concede the general point.
} 
the plausibility of default literal interpretation. ${ }^{9}$ I argue that literal construal without reference change is highly plausible given what we know about verb reference compared to noun reference and activity categorization compared to object categorization. I draw on research in linguistics and psychology to argue that common verbs and activity-concepts are more inclusive than their noun and object-concept counterparts, such that they typically refer to the same activities or capacities even when objects that do or have them differ vastly. We need not determine a priori what it is to do (or have a capacity to do) $\mathrm{X}$ to appreciate that a verb that refers to or is about doing or being able to do $\mathrm{X}$ will satisfy the basic characteristics of activity-referring terms in any language that has this linguistic category-as all human languages do (Haspelmath 2012; Croft 2012; Kemmerer 2014). ${ }^{10}$

In what follows, in Sect. 2 I'll show that the humans-only conviction makes predictions that are inconsistent with what we know about verb semantic hierarchies and verb classes. I then pause to consider some initial responses. These responses help motivate Sect. 3, where I'll provide reasons to think activity categorization typically cross-cuts object domains, such that referential stability of verbs across object domains is the norm. These arguments provide strong reasons for default literal interpretation of psychological verbs used throughout the biological sciences. They also force the objectors to motivate exempting psychological verbs from the basic characteristics of activity-referring terms and concepts, and to provide an alternative, non-ad hoc, explanation of verb reference and activity categorization that shows why we should not take these uses at face value. ${ }^{11}$

\section{Differences in noun and verb patterns of use}

As the organizing elements of sentences, verbs are arguably the most important lexical category in a language; they are certainly the most complex semantically (Miller and Johnson-Laird 1976, p. 523; Brown and Palmer 2010; Gentner and France 1988; Miller and Fellbaum 1991; Gentner and Boroditsky 2001, 2009). In this section I'll

\footnotetext{
9 Yet another task is to provide a theory of reference for verbs, psychological or otherwise. This is mainly a matter of showing how extant theories deal with verb (activity) reference, given that they are developed with noun (object) reference in mind. While a theory that easily deals with semantic variation (such as relevance-theoretic semantics) is more congenial, I need not be committed to any particular theory.

${ }^{10}$ In what follows, I use "verb" for activity-referring terms and concepts, and "activity" in a broad sense to include processes, actions, events, activities, performances, states, and other occurrents, as well as any associated dispositions or capacities to engage in them. Reference to particular events in a familiar philosophical sense, such as Sebastian's stroll at time t, presupposes the reference of verbs (in this case, strolling); the same holds for theories of event individuation (e.g., stabbing and killing, in the question of how Brutus' stabbing Caesar is related to his killing Caesar). I include states as activities because they are being reconceptualized in cognitive science in dynamical terms (e.g., Roediger et al. 2007); their ontological status is also questionable on general grounds (Steward 2012).

11 It bears emphasizing that this foray into linguistics is prompted by the objections to the literal view. Two anonymous reviewers remarked on an earlier draft that (in the words of one) I was "aiming to throw light on which nonhuman organisms have cognitive properties by a linguistic examination of the properties of words for these properties. ... Surely, one thinks, light on this question must come from the science of those organisms, primarily cognitive ethology. How could linguistics help?" My position just is that these sciences are telling us that neurons prefer, etc. The linguistics is for my opponents.
} 
raise some prima facie inconsistencies between the patterns of psychological verb use that the humans-only conviction predicts and the patterns we see. I am not arguing that the way the terms are used is how they should be used, if only because how terms are used does not determine how they should be. ${ }^{12}$ My argument is that many common terms in folk psychology are not only about the folk. So we can be very wrong about human psychological capacities and refer to those capacities using terms that are not restricted to humans. ${ }^{13}$

In linguistic terms, the conviction that psychological verbs are restricted in their (literal) uses for ascribing capacities to humans is the claim that these verbs can only take humans as arguments (mainly in the subject place). Informally, an argument is a slot that is filled by a noun or other syntactic item to yield a sentence; verbs generally take one, two, or three arguments, and their semantics can restrict the kinds of items that fill the slots in literal use. Selectional restrictions on arguments can of course be lexicalized into (that is, included in the conventional meaning of) verbs. Pirouetting is something ballet dancers do; hammering specifies hitting with a hammer; moo selects the argument cows. When non-humans are said to pirouette (e.g., "The earth pirouetted around the sun": Camp 2006), such uses may naturally be interpreted as metaphorical due to these semantic restrictions: planets don't really pirouette because they aren't really ballet dancers. But planets really rotate, as do ballet dancers whenever they pirouette. ${ }^{14}$ In other words, psychological verbs should exhibit patterns of use similar to pirouette, not rotate, if the humans-only conviction was true. Linguistic data show they do not.

\footnotetext{
12 In fact, the idea that future use is so determined is exemplified by Bennett and Hacker's (op.cit.) argument that these uses are nonsense. With Wilson (2006), I agree that the question itself implies a mistaken view of language. Wilson argues that natural languages are fluid and change their "patches" of correct application constantly without anyone taking any notice; we tolerate a lot of openness in how language is used. A relevant case in point is "neuron", which in the original Greek referred to vegetal fibers and was generalized to include all natural fibers (Brenner et al. 2007). Wimsatt's (op.cit.) work emphasizing the messiness of biology can be thought of as the 'material mode' complement to Wilson. As a result, we cannot reliably infer now which extensions of concepts (or terms expressing them) will be permissible in any possible situation. The claim defended in Sect. 3-that verb reference often remains constant across "patches"-is consistent with but independent of Wilson's view (at least on some interpretations).

13 This puts me in opposition to Churchland (1981) even if he is right that we are thoroughly confused about the psychology of the folk: it follows from my view that many "folk"-psychological concepts will figure in a scientific psychology of the folk (and of other things) simply because there is nothing specifically "folk" about many of them. The fact that these terms are associated with a certain amount of human-specific baggage, at least some of which may be added or emphasized in philosophical contexts, is not an argument that this baggage is essential to them; the linguistic and cognitive data discussed in this paper constitute an argument for why it is not essential.

14 As a general pattern, the verb provides a literal organizing element within metaphor. For example, in the noun metaphor "Our piglet is getting dirty", said by a husband to his wife regarding their child playing in mud (Bezuidenhout 2001), the child is metaphorically a piglet but is literally getting dirty. Similarly, Trewavas (2007) agrees with Alpi et al. (2007) that talk of plant "neurobiology" is a metaphor because plants don't have neurons, but he defends the utility of the noun metaphor by arguing that the relevant plant structures have similar information-carrying functions as neurons and other brain structures in animals with brains (see also Brenner et al. 2007, p. 413). Verb metaphors are easily created by choosing verbs that, like pirouette, lexicalize object-specific information or select specific arguments, and then pairing them with arguments that pick out different objects than the ones they select for.
} 
First, the conviction predicts that psychological verbs should be represented disproportionately at low levels in semantic hierarchies, used infrequently, and prompt metaphorical interpretation when used in non-human contexts. This is not what we find. Semantic entailments exemplified by the rotating — pirouetting relation are called troponymy (Fellbaum and Miller 1990; Fellbaum 1990; Miller and Fellbaum 1991). Troponymy relates hyponyms (a.k.a. subordinates or troponyms) to their hyperonyms (a.k.a. superordinates) by kinds of elaborations: manner, intensity, speed, degree of force, argument (object) properties, syntactic roles (subject/object), intention for communicating, epistemic status, ritualization, and more. The general form of the troponymy relation is "To V1 (/V1-ing) is to V2 (/is V2-ing) in some particular manner" ("manner" referring here to any of the modifications). Pirouette is a troponym of rotate, munch of eat, covet of want, and so on. Troponymy relations also form semantic hierarchies of verbs parallel to hierarchies formed by set-membership relations between nouns. ${ }^{15}$ But in contrast with noun hierarchies, every verb hierarchy exhibits a bulge of lexicalized words with rich troponymy relations, and verb hierarchies are much flatter: in the computational lexicon WordNet, the number of levels for verbs rarely exceeds four, compared to 15 or more for nouns (Brown and Palmer 2010; Fellbaum 1990). A comparison of the noun and verb hierarchies formed by "is-a-kind-of" relations (set-membership or troponymy) is represented (roughly) in Fig. 1 (see next page).

In this diagram, nouns (the blue striped triangle) form a many-layered hierarchy of items related by set-membership. ${ }^{16}$ Verbs (the red checkerboard diamond) have a shallow hierarchy (here with four levels, L-1 to L+2) related by troponymy. The figures' relative sizes represent the relative numbers of each word type in a word corpus, and their distinct shapes their different frequency proportions in a corpus (Kucera and Francis 1967). ${ }^{17}$ The triangle's wide base represents the many nouns with low frequency of use (a classic open-class distribution), while the tapering diamond represents few verbs in low-frequency ranges (a semi-closed-class distribution). Finally, the diamond's bulge at $\mathrm{L} 0$ represents the level of many troponyms. For example, stroll, limp, and amble are troponyms of walk, at $\mathrm{L}+1$, which in turn is a subordinate of move (travel) at $\mathrm{L}+2$. Verbs

\footnotetext{
15 Although we use "is a kind of" to refer to set-membership and troponymy relations, they are not the same. Troponymy involves temporal co-extensiveness (pirouette; rotate); activities that are temporally related without troponymy are part-whole (snore; sleep) (Fellbaum 1990). Thus the same activity can be both a superordinate and a part of an activity, which is not true of object kinds: moving is a superordinate of jogging, and a part of jogging (e.g., pushing off with a foot) is also moving. Also, while in principle "troponym" is relative to a level in a semantic hierarchy (as are its customary synonyms "hyponym" or "subordinate"), it is typically used for verbs at level L0 (discussed below). Finally, the once-popular program of organizing verbs by decomposition into semantic atoms (e.g. kill into cause to become not alive) has been overtaken by the semantic organization described here, which takes them as primitive units. Miller and Fellbaum op.cit., pp. 214-215 discuss some reasons for the shift.

16 Note that the noun hierarchy is of count nouns (e.g., kangaroo), while the verb hierarchy is of masslike terms (e.g., jumping or jump). Following Crowther (2011), Hornsby (2012) and Steward (2012), these processes or activities are individualized in context as events (e.g., a jump), analogous to bits of stuffs (e.g. a lump of gold).

17 While Kucera and Frances (op.cit.) remains widely cited, Brysbaert and New (2009) show that the textual sources of words and other corpus features affects word frequency measures. However they do not report different noun/verb frequency proportions or other differences. Fellbaum (1990) reports that the Collins English Dictionary lists 43,636 nouns (averaging 1.74 senses each) and 14,190 verbs (averaging 2.11 senses each).
} 


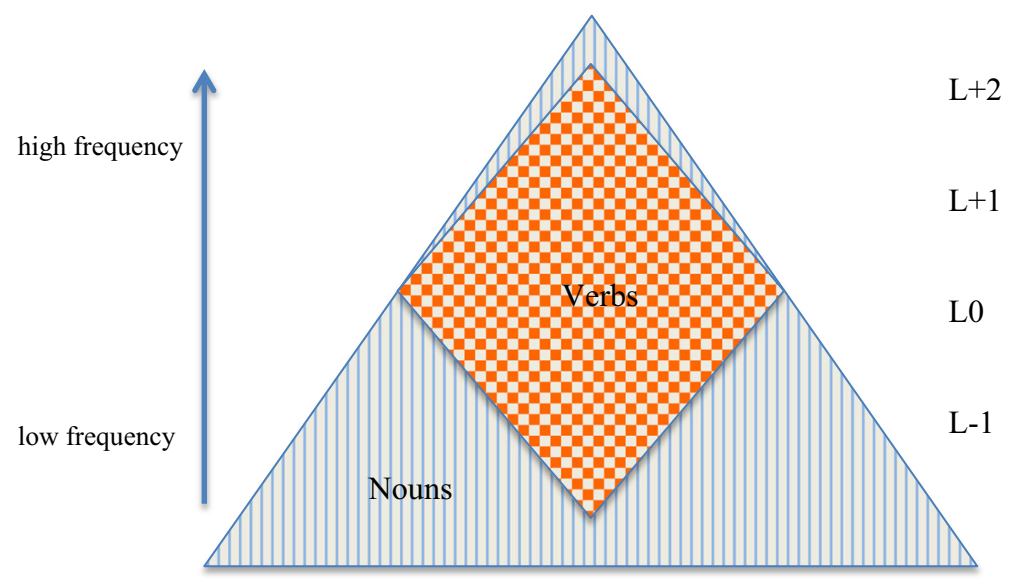

Fig. 1 A visualized comparison of nouns (blue, vertical-striped triangle) and verbs (red, checkerboardpatterned diamond) in terms of hierarchies formed by set-membership or troponymy, frequency of use, and frequency distribution. The levels ( $\mathrm{L} 0, \mathrm{~L}+1$, etc.) indicate verb hierarchy levels; the (many more) levels in the noun hierarchy are not shown. (See text for discussion.). (Color figure online)

at lower levels tend to have more argument restrictions. For example, verbs referring to highly specific activities in sports or skilled work (e.g. jumaring, chamfering) are disproportionately represented at $\mathrm{L}-1$; pirouette is plausibly at $\mathrm{L}-1$, rotate $\mathrm{L} 0$, turn at $\mathrm{L}+1$, and move (make a movement) at L+2. Similarly, communicate, talk, and whisper, or like, love, and idolize, exhibit increasingly specific manners in which an activity may be performed, or emotional responses felt, and thus increasing specificity of possible arguments.

However, to focus on one example, WordNet lists several related senses (polysemes) for the verb prefer: as a verb of emotion (like better; direct hyperonym or superordinate: like), which is the most frequent sense; of cognition (opt between alternatives; direct hyperonym: choose), which is a close second in frequency; and of social relations (favor A over B; direct hyperonym: promote) and social/legal relations (give preference to a creditor; direct hyperonym: pay), both of which are infrequent. The two infrequent senses are restricted to humans via lexicalized social relations. The two frequent senses are not. Pursuing either the like or choose superordinates of the two frequent senses through the database, one finds such verbs as desire or want (for like) and see, view, identify, and regard (for choose), among a large number of senses. One can trace routes through the hierarchy that lack explicit restrictions to humans or human social relations. This prima facie neutrality about the proper domain of the verb in its most frequent senses explains why sentences (1) and (2) (reproduced here) certainly seem on a par:

(1) Four-month-olds prefer looking at motion displays that resemble human walking compared with displays simulating random motion (Bertenthal 1993).

(2) A resonator neuron prefers inputs having frequencies that resonate with the frequency of its subthreshold oscillations (Izhikevich 2007). 
It also explains why these sentences do not cry out for metaphorical interpretation, as does pirouette in "The earth pirouetted around the sun", as the humans-only conviction predicts they should.

Second, the conviction predicts we should have psychological verb classes sharply segregated from other classes in verb classifications. We should not see psychological verbs mixed with non-psychological verbs in a class, and in psychological verb classes we should not see verbs used for non-humans (or if they are they are clearly metaphorical if so used). In one of the most widely used classification systems, Levin (1993) grouped over 3,000 verbs by allowable syntactic alternations (transformations), on the basis that the syntactic regularities would also reflect semantic similarity and coherence. ${ }^{18}$ If psychological verbs were restricted to humans we should also see them forming a clear subclass in the way pirouette, relevé, and so on form a subset of terms subordinate to rotate, rise, and so forth. However, many Levin classes contain both psychological and non-psychological verbs, with no clear argument restrictions to humans. Class 29 (Verbs with Predicative Complements) includes mark and want in the subclass Appoint; the Declare subclass includes (e.g.) judge, think, believe, and declare, while the Dub subclass includes (e.g.) anoint, label, dub and decree. Class 30 (Verbs of Perception) includes detect, sense and see in the See subclass and recognize, perceive, scan, and inspect in the Sight subclass. Class 31 (Verbs of Psychological State) includes verbs that have two arguments, of which one must be an experiencer and the other a stimulus or target, but these include amuse, arouse, distract, perturb, refresh, provoke, startle, stimulate, and threaten - that is, verbs frequently used with non-human arguments. In VerbNet 3.2, these "psych verbs" are divided into the Admire class (Experiencer-Verb-Stimulus frames, e.g. "The tourist admired the painting") and the Amuse class (Stimulus-Verb-Experiencer frames, e.g., "The funny noise amused the children"). Prefer is in the Admire class; decide and communicate, however, are in the Correspond class (which have Agent-Verb frames, e.g. "They agreed about whether he should go.") Depending on different senses, believe is in the Admire and Conjecture classes, Desire in the Want class, Intend in the Try and Wish classes, and Know in the Characterize and Conjecture classes. ${ }^{19}$

Obviously, an objector might respond that there are subclasses of psychological verbs-(e.g.) the Declare subclass forms a coherent semantic class, there is a class of Verbs of Psychological State, and so on. While the class labels themselves are convenient headings, the thrust of the response is that at least some such groups are semantically coherent because the verbs in them denote capacities that only humans

\footnotetext{
18 Using 79 permissible alternations, Levin classified 3,024 verbs (with a total of 4,186 senses) into 48 coarse-grained and 192 fine-grained classes (verbs of motion, communication, etc.) that also exhibit semantic relatedness, including in their possible arguments. VerbNet (http://verbs.colorado.edu/ mpalmer/projects/ verbnet.html) expands on Levin's taxonomy with more verbs, classes, and complementation structures (Fellbaum 1990; Kipper et al. 2008). It has been integrated into the general lexicon WordNet (http:// wordnet.princeton.edu), which contains 15 broad verb files grouped by semantic criteria (e.g. verbs of Bodily Functions and Care, Change, Communication, Cognition, Motion, Perception, etc.). In Sect. 3, I discuss research showing that identifying instances of paradigmatic psychological verbs (think, know) requires knowing syntax.

19 I thank Martha Palmer for alerting me to the need to emphasize that the class of verbs that linguists classify under the label "psychological" is much narrower than the class picked out in folk psychology.
} 
can (really) perform. However, even if this is in fact the best explanation of the semantic coherence of the Declare subclass (or similar subclasses), at best this shows that we can and do sometimes lexicalize human-specific information into psychological verbs, as we do with verbs in any class (e.g., motion verbs). But the appearance of (folk) psychological verbs across classes shows that this does not happen across the board. We should not see this integration if humans-only restrictions were a standard feature of psychological verbs.

Moreover, while this prediction, like the evidence against it, depends on some folkbased notion of a psychological verb, the lack of a precise definition tends to undermine the objector's position. The Anti-Exceptionalist can agree there are subclasses of verbs restricted to humans (as are, e.g., ballet verbs) and that we can agree are psychological. The problem arises when we look at the classes to which the subclasses belong. The broad class provides the intuitive reason for why the subclass is psychological, but the subclass provides its own intuitive reason for why it is humans-only. Perhaps covet is strictly human; is want? Perhaps idolize is strictly human; is like? Since Anti-Exceptionalism doesn't predict that psychological verbs will be meaningfully segregated by humans-only argument restrictions, this lack of intuitively clear borders is not a problem for her. ${ }^{20}$

To summarize this section so far: I've used linguistic data to show that verbs as a word class exhibit patterns of use that support the claim that many psychological verbs will not be human-specific in their literal (referential) uses. As a result, we are entitled, as the default, to take science at its empirically-defeasible word when it tells us that neurons prefer, plants decide, and so forth. These scientists are using psychological verbs in the truth-apt way to pick out properties that are instantiated more widely than many have thought, and about which their research can help us learn more.

But the objector may insist that even if the terms are literal in the new domains, they are now ambiguous. Just because a term is used literally in a new domain, it does not follow that it has the same reference as in the old. When we use cookie in a computer context, we refer to a piece of code from a website that is stored on a computer, not to a sweet baked good. So too with prefers and other psychological terms in non-human domains: in the human case it refers to a psychological capacity, and in the others to something else-perhaps a pattern of behavior, mathematically described.

This response is implausible for both prima facie and deep reasons. The prima facie reason is that a change of reference of this magnitude is often marked in some way, at least initially (such as with scare quotes). Typically there is no such marking. The deep reason is that the claim of reference change lacks independent motivation.

For starters, notice that this response contradicts the metaphor response, which is motivated by a lack of obvious meaning change (assuming, as is usual, that a change in reference entails a change in meaning). A common heuristic for a sharp meaning boundary is the zeugma test (Croft and Cruse 2004, p. 113), illustrated by these sentences:

\footnotetext{
20 From this perspective, Dennett's (1981) ascription of wants, rather than craves or covets, to a lectern to provide an Intentional Stance explanation of its immobility is unsurprising. By the same token, scientists also don't choose any old psychological verb to describe their findings; they choose those verbs that fit the phenomena.
} 
(7) The child had a cookie stored in her backpack.

(8) The computer had a cookie stored in its hard drive.

When sentences such as these are conjoined (perhaps with other syntactic modifications), there is a felt meaning difference, often sensed as a pun. ${ }^{21}$ But the sentence pair (1)-(2) passes the zeugma test (as do examples (3)-(4) (repeated here):

(3) Infants habituated to two jumps of a puppet dishabituated when shown three jumps, and vice versa (Wagner and Carey 2003; Wynn 1996).

(4) The orbitofrontal cortex neurons described here habituate more slowly than is typical of neurons with responses to novel visual stimuli recorded in the amygdala (Rolls et al. 2005).

The objector faces a substantial burden of proof to motivate, non-question-beggingly, why we should think there is a change of reference of the verbs.

The objector might insist that unambiguous literal reference using these verbs is blocked because their semantics entails the possession of capacities that neurons or other non-human entities are not described as having. (In conversation, a frequently mentioned contender is free will.) It could of course turn out that neurons do not prefer because they lack some other distinct capacity that is necessary for having the capacity to prefer. ${ }^{22}$ But legitimate extensions of psychological concepts to new domains cannot be ruled out a priori on the basis of intuitions about what these further capacities might be or which things can have them. The deliverances of such intuitions risk begging the question in two ways: by presupposing that these further capacities cannot also be had by non-humans (when this is a matter of empirical discovery) or by presupposing that only entities that have these further capacities can be truly ascribed the initial capacity now (when the nature of the initial capacity is part of what is in dispute). If it turns out that humans have free will in some sense that neurons do not, and this difference helps explain their ways of preferring stimuli (which is not entailed by this difference), then it is open to us to adjust our language in response, such as by coining a new troponym for human-preferring (a capacity that neurons would not have, just as a planet does not pirouette). Alternatively, we may find a deep similarity, perhaps captured in mathematical models, that humans and neurons share in their preferring; this would yield a deeper understanding of preferring in the way that the discovery of atomic structures deepened our understanding of gold. (We could also discover both, and decide that the similarities outweigh the differences-much as isotopes of oxygen have recognized differences but remain in the extension of "oxygen".) It is also worth noting that scientists can extend individually verbs that may or may not already be embedded in other theoretical frameworks. Whole theories are not built instantaneously; we should not expect theories of cognition to be any different.

\footnotetext{
21 Cruse and Croft's example_- "John and his driver's license expired last Thursday" —is funnier but the point is the same. Ryle use this feature to illustrate category mistakes.

22 For example, it is an open possibility that neural populations, rather than individual neurons, are the proper locus for ascriptions of neural preferences (not unlike Ben-Jacob et al.'s (2012) hypothesis that cancer cell communication is a cooperative activity). This would be an empirical revision consistent with Anti-Exceptionalism.
} 
The next section will intensify the challenge to motivate reference change. I will argue that sameness of reference for activity concepts across object domains is the norm: verbs often have no need to change reference when extended to new domains. ${ }^{23}$

\section{Conceptual categorization of objects and activities}

The claim that many psychological verbs do not have human-specific information lexicalized into them (or that they do not select for human arguments) is, in cognitive terms, the claim that the concepts they express do not encapsulate human-specific information. ${ }^{24}$ In this section I consider what we know or can infer about the information that activity concepts in general $d o$ contain. I will discuss relevant results from work on object categorization, two recent studies of activity naming and categorization, and an influential hypothesis in linguistics posited to explain independent noun/verb differences in acquisition and interpretation. These four sources of evidence converge to indicate that activity concepts typically remain referentially stable across object domains. Referential stability supports a default expectation of the same literal use across domains.

Research on activity categorization-such as the type and amount of information in concepts expressed by verbs at each level of a semantic hierarchy, which level is preferred for categorization, and which activities are prototypes of a given activity category-lags far behind that of nouns and concrete object concepts. This reflects the complexity of verbs, not a lack of interest (Levin 2009, 2012); critical background work on troponymy and verb classes is still fairly recent (e.g., Fellbaum and Miller 1990; Levin 1993). ${ }^{25}$ Verbs are simply harder to organize in semantic hierarchies. However, Fellbaum and Chaffin (1990) provided evidence that verb hierarchies formed by troponymy reflect the organization of the mental lexicon for activity concepts. Since Rosch's and her colleagues' (1973, 1975), Rosch and Mervis (1975), Rosch et al. (1976) studies in concrete object categories were based on information represented by the blue striped triangle in Fig. 1-category levels were drawn from set-membership

\footnotetext{
23 It also seems that they need to not change reference in order to play their roles in sentence organization, object categorization, and metaphor, but I won't defend this here.

24 I set aside here numerous debates about concepts, such as whether they are largely stored in long-term memory or constructed in context (e.g., Croft and Cruse 2004), whether they always contain perceptual or modality-specific information (e.g., Barsalou 2005) and whether they have context-independent common core or overlapping lexicalized constituents (e.g., Carston 2012). I am also neutral regarding various theories of concepts (e.g., prototypes, exemplars, and theories), though adopt prototype theory for ease of exposition. For the same reason, I will also adopt a standard, if not universally held, view by which the meaning of a word is the concept it expresses (hence that verbs express activity concepts) and that concepts have a lexicalized (context-independent) core encoded in memory (which may be semantically elaborated in context). The issue of feature overlap (with no single common feature shared by all members of a category) raises the issue of when one has passed from polysemy to homonymy (when a word form has distinct senses, as with "bank"); this is the sort of boundary issue that the 'zeugma test' and other similar tests are designed to help adjudicate.

25 For example, in one test, participants were asked to generate verbs to a sample verb. They found that is-a-manner-of (troponymy) relations between the sample and generated verbs accounted for $25.1 \%$ of the resulting pairs (entailment accounted for another $14.4 \%$ and synonymy for $13.5 \%$, with the remainder divided among three other relations).
} 
hierarchies of superordinate, basic, and subordinate nouns, and nouns were chosen based on the same frequency data - this means that both kinds of semantic hierarchies modeled in Fig. 1 are valid as a basis for categorization research. That said, despite this lag the research described below provides indirect evidence for referential stability.

The first source is Rosch and her colleagues, who found that basic-level nouns corresponded to categories that maximize similarity between objects in the category and minimize similarity with objects not in the category. The information contained in the object concepts (denoted below in all capital letters) at each level was measured by feature lists, which were elicited from participants in various ways. This measure showed that basic nouns corresponded to categories with the most information about features common to category members and the least information about features shared between category members and members of other categories. But they also found that superordinates had fewer common attributes listed than basic terms, and-significantly in this context-many of these common features were "functional" rather than physical: they involved common behaviors or uses (e.g., "make things" for TOOL, as opposed to "teeth" for SAW). Miller and Fellbaum (1991, p. 207) characterize these "functional" features of nominal concepts in general as "all the features of nominal concepts that are described by verbs or verb concepts". In short, the features that bind superordinate object categories are often common activities. It follows that the verbs commonly used to name these activities are referentially stable across object domains.

Relatedly, children have difficulty learning superordinate categories of objects (Horton and Markman 1980; Rosch et al. 1976). This may be because they must learn "functional" features corresponding to verbs, and children find verbs (even concrete verbs) harder to learn than nouns (Gentner 1981). ${ }^{26}$ (A widely accepted explanation of this difficulty is discussed below.) In addition, object superordinates are often mass terms and children have difficulty learning mass terms (Markman 1985); if verbs pick out masses (see fn. 16), the features common to superordinate object categories will be masses, and so this factor too may contribute to the learning difficulty.

The second source is Tranel et al. (2008), who conducted a neuropsychological study of activity naming. Normal and brain-damaged patients were shown short film clips and pictures and had to name the activity shown by using a verb. In developing their dynamic stimuli, the target list of verbs that determined naming accuracy, drawn from Levin (op.cit.), included such words as bending, cutting, grazing, kissing,

\footnotetext{
26 Rifkin (1985) tested whether Rosch's results could be extended to events, but he used event hierarchies that were not formed by troponymy relations between verbs. He found that subjects listed few features for superordinates ("medical activity", "hygiene") and more and similar numbers of features for basic ("surgery", "shower") and subordinate ("heart surgery", "lockerroom shower") event categories. Pulman (1983) asked participants to list features of activities denoted by verbs at two levels, hyperonym and hyponym, but they often responded by providing synonyms or attempting to give definitions. He found differences in judgments of representativeness among hyponyms in a category (e.g. for walk, stride was most representative, march in the middle, limp least representative among the 8 hyponyms provided; for speak, recite was most, whisper middle, stutter least). Morris and Murphy (1990), using Rifkin's stimuli, found that subordinate events in the same category (subway travel, travel by school bus) were judged more similar than subordinate objects in the same category (rowboat, sports car) and interpreted this result as suggesting that superordinate event categories (transportation) were more informative. But it equally well suggests that judgments of similarity between events (in their sense) require less information. Murphy and Lassaline (1997) review these and similar studies.
} 
parachuting, pushing, swimming, and tip-toeing. After the stimuli were developed, normal participants in the study correctly identified the activity in the film clips with an average $90.8 \%$ accuracy. Since many of the verbs could have been illustrated using a wide range of objects and scenes (e.g. compare pushing, cutting, or bending with parachuting or tip-toeing), this result suggests that many activities are readily categorized under concepts that pick out features common to many object categories. Correct identification of an activity depends on information provided in a particular scene, but preferred categorization of the activity appears to be at a level that is largely independent of the specific scene illustrating the activity.

Thus, while Fellbaum and Chaffin (op.cit., p. 425) suggest that troponyms (e.g., munching as a troponym of eating) are basic in that they carry more information than their superordinates, it doesn't follow that troponyms are preferred in categorization. To the contrary, Fiez and Tranel (1997, p. 556) found that 59 out of 64 subjects used eating to name a relevant pictured action when developing their standardized stimuli for studies in retrieving knowledge of actions (the other, non-standard, choices were tasking (2), anticipating, hungry and snacking). In addition, in their analysis of participants' non-standard names of pictured actions, a far higher percentage of these non-standard names were superordinates (42\%) compared to the percentage of superordinates among the non-standard names provided for objects $(10 \%)$ in a related study of nouns and object concepts.

Of course, Tranel et al.'s and Fiez and Tranel's studies focused on concrete verbs that pick out observable actions. ${ }^{27}$ However, in a study of identifying instances of action, light, and mental verbs, Snedeker and Gleitman (2004, p. 272 passim; see also Gleitman et al. 2005 and fn. 29 below on light verbs) found that reliable (close to $90 \%$ ) identification of psychological verbs (in particular, credal verbs think and know) depends dramatically on syntactic information, such as how many arguments a verb takes. Syntactic information was provided by giving participants sentence frames in which content words were replaced with nonsense (e.g. "Did you play with the elephant?" is transformed into "Did er PILK with the ramermok?", where the capitalized string replaces the verb to be identified). When given only visual (scene) information, or knowledge of the nouns used in a context, or both, reliable identification of these verbs hovered around $10 \%$. In short, reliable identification of instances of paradigmatic non-observable psychological properties requires knowledge of syntax. So psychological verbs do not violate the pattern exemplified by concrete verbs: we can reliably name instances of activities using verbs that do not lexicalize information specific to a particular illustrating object or scene. Sentence frames may restrict possible arguments by (e.g.) specifying an agent in the grammatical subject position, but it does not follow that this must be a human agent. ${ }^{28}$

\footnotetext{
27 Tranel et al.'s list included few psychological verbs (e.g., denying). Similarly, Fiez and Tranel (op.cit.) selected frequently used verbs (from Kucera and Francis op.cit.) that could easily be depicted in photographs (singly or in before/after pairs) and which reliably elicited 1-3 predominant responses from normal subjects. Few of these verbs were psychological (e.g., interviewing (speaking), looking, yelling, praying, and refusing) even though some psychological verbs (e.g., see, say, think, know) are among the most frequent in a corpus.

28 As another example, children are said to possess the concept of belief on the basis of ascribing false beliefs to puppets at an age (about 4) when they don't reliably know that puppets can't have beliefs-either they're not sure puppets can't, or they think puppets could have beliefs by magic (Harris et al. 1991).
} 
The third source is Stamenkovic (2011), who used verb frequency data from the 2011 edition of the Corpus of Contemporary American English (Davies 2008) to investigate prototypicality for activities, rather than objects. Based on their research in object categorization, Rosch and colleagues theorized that concepts are prototypes, unified by overlapping features or family resemblance rather than necessary and sufficient conditions (Rosch and Mervis 1975). Categories form around prototype items that exemplify many of the features that are frequently possessed by members. For example, a robin is a prototype of a bird because it has many of the features frequently listed for this category. If object categories reflect co-occurring clusters of attributes in the world, prototypes are objects that best exhibit the attributes in a given cluster; they are the members of an open category that play normative roles for the category, even though categories have equally real non-prototypical members (e.g., penguins). While prototypicality is a feature of category members - a robin is a prototypical bird; robin is not a bird at all-Stamenkovic's idea was that if a particular kind of activity is more prototypical of an activity class than another, then ceteris paribus a verb used to name the prototypical activity will be more frequently used.

He examined frequency data for verbs from two Levin-based verb classes: Verbs of Motion (around an axis) and Verbs of Desire, want-subclass (I'll call them the Motion and Want classes). The Motion class included turn, twist, spin, whirl, twirl, rotate, revolve and coil; the Want class included want, need, desire, covet, crave, and fancy. By this measure, turn picks out the most prototypical activity in the Motion class, followed by spin and twist, rotate, revolve and whirl, and twirl and coil; while want picks out the most prototypical state in the Want class, followed by need, desire, crave, and fancy and covet. Even if (as may be possible) turn and want are hyperonyms (superordinates) in their respective classes, the remaining verbs' relative frequencies, and hence the prototypicality of the activities or states they pick out, still differed.

In both classes, the verbs appeared intuitively to have more semantic features or more intense presentations of features as one moved from frequent (prototypical) to infrequent: increasing degree and intensity of motion in the Motion class, increasing degree and intensity of emotion in the Want class. This suggests that prototypicality for activities (as measured by greater frequency) is correlated with fewer semantic elements. To be clear, there was no objective measure of semantic complexity or information content in this study, and no control for greater frequency due to more opportunities for use, polysemy, or other confounds. ${ }^{29}$ But even this rough measure

\section{Footnote 28 continued}

The restriction barring puppets from literal ascription of believes is not lexicalized in the verb, as is (in contrast) the restriction barring a planet from literal pirouetting.

29 The informational content in concepts expressed by verbs at different levels of a verb hierarchy remains to be adequately operationalized and measured. However, the idea of an informational difference has been put in terms of a contrast between heavy and light verbs (Brown 2008). Verbs with rich, contextually specific semantics, often incorporating object properties, are heavy. (Heavy nouns may be nouns denoting animate beings (Gentner and Boroditsky 2001, p. 245); proper names may be heaviest of all and therefore atypical of reference in general.) Prototypical light verbs, such as go, do, and get, lexicalize very little, and are often combined with nouns to form contentful wholes (such as go skiing). Feature lists would be a way to operationalize the heavy/light distinction. Fiez and Tranel (op.cit.) designed a test for knowledge of action features in which subjects chose between two pictures of actions to answer 8 comparative attribute questions, such as which action would take longer to complete, make the loudest noise, be most physically 
suggests a difference in categorization in which prototypicality (frequency) correlates with fewer features for activities than objects (less information in the corresponding verbs than nouns). For example, turn is more frequent than rotate, and desire more frequent than crave, even though every rotating is a turning, and every craving a desiring, but not vice versa. In contrast, mammal is less frequent than (and associated with fewer features than) $d o g$, even though every dog is a mammal but not vice versa (and a dog is a prototypical mammal). ${ }^{30}$ This suggests that criteria for prototypicality for activities are less stringent than for objects: there are likely to be fewer typical features overall, an activity prototype may need to exemplify fewer of them to count as a prototype, and activity prototypes are likely to be less similar to each other than object prototypes are to each other. In turn, criteria for belonging to a category picked out by a verb will allow for more variation from the prototype. This cross-domain stability would in turn enable the formation of "function"-heavy superordinate object categories, as well as help explain the different roles of nouns and verbs in metaphor (see fn. 14).

The fourth source is the dominant explanation of a number of well-known noun/verb differences in acquisition, meaning variability, and interpretation, which imply that verbs typically do not lexicalize a lot of information in themselves. ${ }^{31}$ This explanation traces these linguistic and cognitive differences to the differences in their referentsi.e. objects vs. activities. This difference is encapsulated in the psychological literature in Gentner's Natural Partitions (NP) and Relational Relativity (RR) hypotheses (Gentner 1981, 1982, 2006; Gentner and Boroditsky 2001; others, e.g. Talmy 1975 and Rosch et al. (1976), make similar proposals). NP holds that "the noun class has the privilege of naming the highly cohesive parts of the world", whereas "there is no natural individuation for the referents of verbs" (Gentner 2006, p. 544). RR is the complementary claim that verb reference varies more across languages - that "even fairly concrete verb meanings (such as those of motion verbs) make a selection from the

Footnote 29 continued

tiring, etc.. A corresponding test for semantic knowledge substituted words for pictures. However these tests do not operationalize information differences between superordinate and basic terms.

30 Rosch et al. (op.cit.) found that categorization of concrete objects is preferred at the basic level. For example, subjects were quicker to identify an object from visual stimuli when primed by basic rather than superordinate or subordinate nouns. Thus, concepts expressed by basic-level nouns contain the optimal amount and kind of information for classification and prediction, and are preferred in many cognitive tasks. In contrast, superordinate verbs appear to be preferred.

31 Across languages, children find verbs harder to learn than nouns, even though prelinguistic infants are able to distinguish both objects and events, and learn novel actions quicker than either novel nouns or verbs but still learn novel nouns before novel verbs (Gleitman et al. 2005; Childers and Tomasello 2002; Tardif et al. 1999; Gentner and Boroditsky 2001). Verb meaning also varies more than noun meaning, both across contexts and between languages. This semantic flexibility has been suggested to explain the well-known greater polysemy of verbs, as measured by the number of subentries for distinct but related senses in a lexical dictionary (Gentner 1981, p. 169); within-language variability conventionalized as polysemy also affects performance on semantic decision tasks (Brown 2008). Importantly, polysemy is not homonymy (distinct unrelated senses), and can be explained by sameness of reference even if meaning is not (identical to) reference (Rakova 2003). Verb meaning is also more mutable in that we tend to select a verb meaning to fit a relatively fixed noun meaning to obtain overall sense (Gentner 1981). For example, when interpreting the sentence "The crowd was moved by his face", we are more likely to interpret moved in the emotional sense to fit face rather than interpret face to refer to an object that can change the location of a crowd. 
available relational information, and just which information is selected varies across languages" (op.cit., p. 544; by "meanings" here Gentner means referents). To use an example from Talmy (op.cit.), when describing an empty bottle on water going into a cave, English uses a verb that lexicalizes the manner of motion ("The bottle floated into the cave"), Spanish uses a verb that lexicalizes the direction ("La botella entró a la cueva flotando"), and both converge on a common referent by using appropriate modifiers (into and flotando) to complement what each verb lexicalizes on its own to attain overall sentential meaning. In contrast, bottle and botella pick out the same contextindependent referent on their own. ${ }^{32}$ Gentner (1982, pp. 323-324) also describes the referential difference this way:

[D]ifferences in degree of differentiation [e.g. among subspecies of seal in Inuit languages] do not constitute a radically different parsing of the world, any more than would be the presence of more names for different-shaped bottles in Spanish than in English. The basic decision as to which parts of the scene belong to the seal and which to the background is not in question. If Eskimo were found to have a term for, say, the object composed of the head of a seal and the crest of a nearby wave, then this would constitute a case of cross-linguistic variability comparable to that found for predicate terms.

In the philosophical literature, this type of description should be familiar from Hirsch's (1982, 1993) discussion of "strange" languages, in which (e.g.) the state of affairs of a car backing out of a garage can be individuated instead such that what we observe is the gradual coming into being of an outcar and ceasing to exist of an incar (or, to elaborate Talmy's example, the gradual ceasing to exist of an outbottle and gradual coming into being of an inbottle). In these terms, if a language is strange when one of its word classes is strange (relative to the same word class in another language), then human languages are already strange relative to each other because of their verbs. Cross-linguistically, what is strange for nouns is normal for verbs (and NP explains why this is strange for nouns).

It follows from this referential difference that verbs naturally enjoy degrees of referential freedom that are unavailable for nouns. More accurately, our languages and conceptual schemes concentrate in their verbs the referential degrees of freedom that there are. Hirsch shows that this division of referential labor is not metaphysically given, but linguists show that it is empirically robust. But verbal referential flexibility is possible if less information is lexicalized into verbs to begin with. In contrast to float and entrar, if lo in Tzeltal picks out eating soft things, it cannot be used (literally) to pick out an eating of a carrot even though the extensions of eating and lo already overlap in a way that float and entrar do not. We can't take the soft out of lo any

\footnotetext{
32 Similarly, Korean has a common verb (kkita) lexicalizing or encoding tightness of fit, cross-cutting the English verbs for putting in and putting on (Bowerman and Choi 2003); Cantonese lexicalizes epistemic status or evidentiality into a verb translatable as falsely-believe, while English uses a separate modifier (Cheung et al. 2009); Tzeltal contains specific eat-verbs for eating different kinds of things: we (eat tortilla or bread), k'ux (eat crunchy things), lo (eat soft things) and ti (eat meat or bite) (Brown 2008). This variability is also psychologically real. Cheung et al. (op. cit., p. 153) found that Cantonese-speaking children exhibited false-belief understanding when the evidential status was lexicalized in the verb, but not if it was conveyed by a separate particle.
} 
more than we can take the ballet dancer out of pirouette. In fact, a bias to assume that the relative information-richness of nouns is the norm would explain why two of the main objections to Anti-Exceptionalism involve reference change or metaphor: these responses presuppose a clash between the features conventionally picked out by a term and the presence of those very features (or many of them) in a new context.

Based on this research, the referential stability hypothesis seems to hold for many common verbs, and ipso facto for many common psychological verbs. Superordinate categories of objects are often bound by activities that are common across object domains. Activities that can be performed by many different kinds of objects are easily identifiable from static or dynamic visual stimuli, and while visual information is insufficient for enabling identification of instances of credal or other paradigmatically mental verbs, adding syntactic information-in particular, sentence frames providing information about argument places-does the trick. By one rough measure based on verb frequency, prototypical activities have fewer features compared to prototypical objects. Finally, an independent explanation of other noun/verb differences implies that common verbs do not lexicalize as much information as common nouns; coextensiveness of verbs across languages requires the semantic contributions of modifiers. As a result, we are entitled to assume, as the default, that the capacities many common psychological concepts categorize are not human-specific. In consequence, we are entitled to assume, as the default, that their use in non-human domains in serious scientific contexts are reports of new discoveries, as they seem.

\section{Concluding remarks}

A comprehensive theoretical framework appropriate to verbs and activity concepts, to which psychological verbs and concepts belong, provides ample reason to assume a default literal interpretation when psychological verbs are used in non-psychological biological sciences. This evidence from linguistics and psychology strongly suggests that verbs are referentially robust across many object domains and extend easily to new domains without varying in reference. As a result, when scientists use these terms to report new findings, we are entitled to take them at their word.

Although we associate many human characteristics with psychological capacities, the general pattern of verb use and activity categorization mitigates against thinking that these associations are essential to the capacity rather than contingent features of their instantiation by (normal, adult) humans. This would explain why psychological verbs are so readily available for use in non-human domains to begin with. Rather than think such uses are cognitively defective, it is more plausible that they pick out categories of which some of their human manifestations are familiar prototypes, but which have equally real, as-yet non-prototypical, members. We stand to be able to understand psychological capacities better by seeing the full range of ways in which they can be possessed and by developing formal models of them that are applicable across many domains.

This is not to deny that psychological verbs differ in important respects from other verbs. As noted, they are even more difficult to learn than other verbs, and accurate identification of instances requires linguistic (syntactic) as well as perceptual informa- 
tion (Gleitman et al. 2005; Cheung et al. 2009). This does not restrict them to humans, however. It is also an important syntactic feature of many psychological verbs that they take sentential complements, but they are not unique in doing so (Gleitman et al. 2005; Snedeker and Gleitman 2004). Sentential complements need not be expressed by that-clauses- "Success requires working long hours", "I needed to come" include sentential complements (VerbNet) — and verbs taking such complements (requires) need not be psychological. The propositional contents expressed in the sentential complements of psychological verbs when they are ascribed to humans are also specific to humans and their social relations; they may or may not be language-dependent, as Davidson (op.cit.) argued. But this does not entail that the verbs pick out a capacity (an "attitude") that only humans can possess; the specification of a propositional content may instead yield a kind of troponym. (This constitutes a form of adverbialism.) Finally, psychological verbs are different from other verbs because humans cherish and self-identify with the capacities to which they refer, establish social rankings based on these capacities, and deprive others of them in order to aggress. But these special roles they play in our lives are not reasons to think they are special to us in a metaphysical sense.

The debate over literal use may seem esoteric, but it determines our attitude towards the findings of biological sciences. What these sciences do not need at this stage of inquiry is a theoretically crippling (and question-begging) assumption that the human cases are the only real cases of these capacities. Many of us already accept the basic idea that the same psychological capacity can be realized by something else in a somewhat different way, such as in vision and feeling pain, even if we can't experience what other entities see or feel. We have good reasons to generalize this basic idea.

Acknowledgments I would like to thank participants in the June 2013 "What is Cognition?" workshop at Ruhr Universitat - Bochum, in partciular Colin Allen, Albert Newen, and co-organizers Cameron Buckner and Ellen Fridland, for helpful responses to my initial attempts at working out some of these issues; and to the University of Pittsburgh's Center for Philosophy of Science, in particular John Norton, Jim Bogen, Mazviita Chirimuuta, and my fellow fellows, for further questions and encouragement. I also thank my colleague Evan Fales for providing examples of verbs that refer to very specific activities in rock-climbing and woodworking. I also am deeply grateful to three (I think) anonymous reviewers for detailed critical comments on a previous draft, which led to enormous improvements and clarifications throughout.

\section{References}

Alpi, A., Amrhein, M., Bertl, A., Blatt, M., Blumwald, E., Cervone, F., et al. (2007). Plant neurobiology: No brain, no gain? Trends in Plant Sciences, 12(7), 135-136.

Andrews, K. (2009). Politics or Metaphysics? On attributing psychological properties to animals. Biology and Philosophy, 24(1), 51-63.

Andrews, K. (2014). Animal cognition. Stanford Encyclopedia of Philosophy (Fall 2014 edition). E. Zalta, ed., http://plato.stanford.edu/archives/fall2014/entries/cognition-animal/.

Barsalou, L. W. (2005). Situated conceptualization. In H. Cohen \& C. Lefebvre (Eds.), Handbook of categorization in cognitive science (pp. 619-650). St. Louis: Elsevier.

Bekoff, M., Allen, C., \& Burkhardt, G. (2002). The cognitive animal: Empirical and theoretical perspectives on animal cognition. Cambridge, MA: MIT Press.

Ben-Jacob, E., Coffey, D., \& Levine, H. (2012). Bacterial survival strategies suggest rethinking cancer cooperativity. Trends in Microbiology, 20(9), 403-410.

Bennett, M. R., \& Hacker, P. M. S. (2003). Philosophical foundations of neuroscience. Malden, MA: Blackwell Publishing Ltd. 
Bennett, M., Dennett, D., Hacker, P., \& Searle, J. (2007). Neuroscience \& philosophy: Brain, mind, \& language. New York: Columbia University Press.

Bermudez, J. L. (2003). Thinking without words. Cambridge, MA: MIT Press.

Bertenthal, B. I. (1993). Perception of biomechanical motions by infants: Intrinsic image and knowledgebased constraints. In C. Granrud (Ed.), Carnegie symposium on cognition: Visual perception and cognition in infancy (pp. 175-214). Hillsdale, NJ: Lawrence Erlbaum.

Bezuidenhout, A. (2001). Metaphor and what is said: A defense of a direct-expression view of metaphor. Midwest Studies in Philosophy, XXV, 156-186.

Bowerman, M., \& Choi, S. (2003). Space under construction: Language-specific spatial categorization in first-language acquisition. In D. Gentner \& S. Goldin-Meadow (Eds.), Language in mind: Advances in the study of language and thought (pp. 387-427). Cambridge, MA: MIT Press.

Brenner, E. D., Stahlberg, R., Mancuso, S., Vivanco, J., Baluška, F., \& Von Volkenburgh, E. (2006). Plant neurobiology: An integrated view of plant signaling. Trends in Plant Sciences, 11, 413-419.

Brenner, E. D., Stahlberg, R., Mancuso, S., Baluška, F., \& Von Volkenburgh, E. (2007). Reply to Alpi et al.: Plant neurobiology: The gain is more than the name. Trends in Plant Sciences, 12(7), 285-286.

Brown, S. W. (2008). Polysemy in the mental lexicon. Colorado Research in Linguistics, 21, 1-12.

Brown, S. W., \& Palmer, M. (2010). What computers need to know about verbs. In P. Sheu, H. Yu, C. V. Ramamoorthy, A. K. Joshi, \& L. A. Zadeh (Eds.), Semantic computing (pp. 13-31). New York: Wiley-IEEE Press.

Brysbaert, M., \& New, B. (2009). Moving beyond Kucera and Francis: A critical evaluation of current word frequency norms and the introduction of a new and improved word frequency measure for American English. Behavior Research Methods, 41(4), 977-990.

Calvo, P., \& Keijzer, F. (2009). Cognition in plants. In F. Baluška (Ed.), Plant- environment interactions: Signaling and communication in plants (pp. 247-266). Berlin: Springer-Verlag.

Camp, E. (2006). Metaphor in mind: The cognition of metaphor. Philosophy Compass, 1(2), 154-170.

Carston, R. (2012). Metaphor and the literal/nonliteral distinction. In K. Allan \& K. Jaczszolt (Eds.), Cambridge handbook of pragmatics (pp. 469-492). Cambridge: Cambridge University Press.

Cheung, H., Chen, H.-C., \& Yeung, W. (2009). Relations between mental verb and false-belief understanding in cantonese-speaking children. Journal of Experimental Child Psychology, 104, 141-155.

Childers, J. B., \& Tomasello, M. (2002). Two-year-olds learn novel nouns, verbs, and conventional actions from massed or distributed exposures. Developmental Psychology, 38(6), 967-978.

Churchland, P. (1981). Eliminative materialism and the propositional attitudes. The Journal of Philosophy, $78(2), 67-90$.

Croft, W. A. (2012). Verbs: Aspect and causal structure. Oxford: Oxford University Press.

Croft, W. A., \& Cruse, D. A. (2004). Cognitive linguistics. Cambridge, UK: Cambridge University Press.

Crowther, T. (2011). The matter of events. The Review of Metaphysics, 65, 3-39.

DasGupta, S., Ferreira, C., \& Miesenböck, G. (2014). FoxP influences the speed and accuracy of a perceptual decision in Drosophila. Science, 344, 901-904.

Davidson, D. (1975). Thought and talk. In S. Guttenplan (Ed.), Mind and language. New York: Oxford University Press.

Davies, M. (2008). The corpus of contemporary American English: 450 million words, 1990-present. http:// corpus.byu.edu/coca/.

Dennett, D. (1981). True believers: The intentional strategy and why it works. In A. F. Heath (Ed.), Scientific explanation: Papers based on Herbert Spencer lectures given in the University of Oxford (pp. 150167). Oxford: Clarendon Press.

Fellbaum, C. (1990). English verbs as a semantic net. International Journal of Lexicography, 3(4), $278-301$.

Fellbaum, C., \& Chaffin, R. (1990). Some principles of organization of verbs in the mental lexicon (pp. 420-427). Program of the 12th Annual Meeting of the Cognitive Science Society.

Fellbaum, C., \& Miller, G. A. (1990). Folk psychology or semantic entailment? A reply to Rips and Conrad (1989). Psychological Review, 97(4), 565-570.

Fiez, J., \& Tranel, D. (1997). Standardized stimuli and procedures for investigating the retrieval of lexical and conceptual knowledge for actions. Memory \& Cognition, 25(4), 543-569.

Gentner, D. (1981). Some interesting differences between nouns and verbs. Cognition and Brain Theory, $4(2), 161-178$.

Gentner, D. (1982). Why nouns are learned before verbs: Linguistic relativity versus natural partitioning. In S. Kuczaj (Ed.), Language development: language, cognition, and culture (pp. 301-334). Hillsdale, NJ: Erlbaum. 
Gentner, D. (2006). Why verbs are hard to learn. In K. Hirsh-Pasek \& R. Golinkoff (Eds.), Action meets word: How children learn verbs (pp. 544-564). Oxford: Oxford University Press.

Gentner, D., \& Boroditsky, L. (2001). Individuation, relativity and early word learning. In M. Bowerman \& S. Levinson (Eds.), Language acquisition and conceptual development. New York: Cambridge University Press.

Gentner, D., \& Boroditsky, L. (2009). Early acquisition of nouns and verbs: Evidence from Nav. In V. C. Mueller Gathercole (Ed.), Routes to language: Essays in honor of Melissa Bowerman (pp. 5-35). New York: Psychology Press.

Gentner, D., \& France, I. M. (1988). The verb mutability effect: Studies of the combinatorial semantics of nouns and verbs. In S. L. Small, G. W. Cottrell \& M. K. Tanenhaus (Eds.), Lexical ambiguity resolution: Perspective from psycholinguistics, neuropsychology and artificial intelligence (pp. 343382). San Mateo, CA: Morgan Kaufmann Publishers, Inc.

Gleitman, L. R., Cassidy, K., Papafragou, A., Nappa, R., \& Trueswell, J. T. (2005). Hard words. Journal of Language Learning and Development, 1, 23-64.

Harris, P., Brown, E., Marriott, C., Whittall, S., \& Harmer, S. (1991). Monsters, ghosts, and witches: Testing the limits of the fantasy-reality distinction in young children. British Journal of Developmental Psychology, 9, 105-123.

Haspelmath, M. (2012). How to compare major word-classes across the world's languages. UCLA Working Papers in Linguistics, Theories of Everything, 17(16), 109-130.

Hirsch, E. (1982). The concept of identity. New York: Oxford University Press.

Hirsch, E. (1993). Dividing reality. New York: Oxford University Press.

Hornsby, J. (2012). Actions and activity. Philosophical Issues, 22(1), 233-245.

Horton, M., \& Markman, E. (1980). Developmental differences in the acquisition of basic and superordinate categories. Child Development, 51(3), 708-719.

Hubel, D. H., \& Wiesel, T. N. (1962). Receptive fields, binocular interaction and functional architecture in the cat's visual cortex. Journal of Physiology, 160, 106-154.

Izhikevich, E. M. (2007). Dynamical systems in neuroscience: The geometry of excitability and bursting. Cambridge, MA: MIT Press.

Johnson, C. (1997). Metaphor vs. conflation in the acquisition of polysemy: The case of see. In Cultural, psychological, and typological issues in cognitive linguistics (pp. 155-69).

Keeley, B. L. (2004). Anthropomorphism, primatomorphism, mammalomorphism. Philosophy \& Biology, $19,521-540$.

Keller, E. F. (1983). Feeling for the organism: The life and work of Barbara McClintock. New York: W.H. Freeman \& Co.

Kemmerer, D. (2014). Word classes in the brain: Implications of linguistic typology for cognitive neuroscience. Cortex, 58, 27-51.

Kipper, K., Korhonen, A., Ryant, N., \& Palmer, M. (2008). A large-scale classification of English verbs. Language Resources and Evaluation Journal, 42(1), 21-40.

Kucera, H., \& Francis, W. (1967). Computational analysis of present-day American English. Providence, RI: Brown University Press.

Levin, B. (1993). English verb classes and alternations. Chicago: University of Chicago Press.

Levin, B. (2009). Where verb classes come from. Presentation notes for Verb Typologies Revisited, Ghent, Belgium, Feb 5-7, 2009.

Levin, B. (2012). Manner and result: Implications for Argument realization across languages. Presentation notes for Dusseldorf, July 5, 2012.

Lyon, P. (2006). The biogenic approach to cognition. Cognitive Processing, 7, 11-29.

Markman, E. (1985). Why superordinate category terms can be mass nouns. Cognition, 19(1), 31-53.

Miller, G. A., \& Fellbaum, C. (1991). Semantic networks of English. Cognition, 41, 197-229.

Miller, G. A., \& Johnson-Laird, P. N. (1976). Language and perception. Cambridge, MA: Harvard University Press.

Morris, M. W., \& Murphy, G. L. (1990). Converging operations on a basic level in event taxonomies. Memory \& Cognition, 18, 407-418.

Murphy, G. L., \& Lassaline, M. E. (1997). Hierarchical structure in concepts and the basic level of categorization. In K. Lamberts \& D. Shanks (Eds.), Knowledge, concepts and categories (pp. 93-132). Hove: Psychology Press.

O'Keefe, J., \& Dostrovsky, J. (1971). The hippocampus as a spatial map. Preliminary evidence from unit activity in the freely-moving rat. Brain Research, 34, 171-175. 
ojalehto, B., Waxman, S. R., \& Medin, D. L. (2013). Teleological reasoning about nature: Intentional design or relational perspectives? Trends in Cognitive Sciences, 17(4), 166-171.

Pulman, S. G. (1983). Word meaning and belief. London: Croom Helm.

Rakova, M. (2003). The extent of the literal: Metaphor, polysemy and theories of concepts. Houndmills: Palgrave-MacMillan.

Rifkin, A. (1985). Evidence for a basic level in event taxonomies. Memory \& Cognition, 13(6), 538-556.

Rips, L. J., \& Conrad, F. G. (1989). Folk psychology of mental activities. Psychological Review, 96, 187207.

Rips, L. J., \& Conrad, F. G. (1990). Parts of activities: Reply to Fellbaum and Miller (1990). Psychological Review, 97(4), 571-575.

Roediger, III., Henry, L., Dudai, Y., \& Fitzpatrick, S. M. (2007). Science of memory: Concepts. Oxford: Oxford University Press.

Rolls, E. T., Browning, A. S., Inoue, K., \& Hernadi, I. (2005). Novel visual stimuli activate a population of neurons in primate orbitofrontal cortex. Neurobiology of Learning and Memory, 84, 111-123.

Rosch, E. (1973). Natural categories. Cognitive Psychology, 4, 328-350.

Rosch, E. (1975). Cognitive Representations of semantic categories. Journal of Experimental Psychology, 104(3), 192-233.

Rosch, E., \& Mervis, C. (1975). Family resemblances: Studies in the internal structure of categories. Cognitive Psychology, 7, 573-605.

Rosch, E., Mervis, C. B., Gray, W. D., Johnson, D. M., \& Boyes-Braem, P. (1976). Basic objects in natural categories. Cognitive Psychology, 8, 382-439.

Schwitzgebel, E. (2012). A dispositional approach to the attitudes: Thinking Outside the belief box. (MS).

Slaney, K., \& Maraun, M. (2005). Analogy and metaphor running amok: An examination of the use of explanatory devices in neuroscience. Journal of Theoretical and Philosophical Psychology, 25(2), $153-172$.

Snedeker, J., \& Gleitman, L. (2004). Why it is hard to label our concepts. In D. Hall \& S. R. Waxman (Eds.), Weaving a lexicon. ambridge, MA: MIT Press.

Sober, E. (2005). Comparative psychology meets evolutionary biology: Morgan's canon and cladistic parsimony. In L. Datson \& G. Mitman (Eds.), Thinking with animals: New perspectives on anthropomorphism (pp. 85-99). New York: Columbia University Press.

Stamenkovic, D. (2011). Verbs and prototype theory: State of the art and possibilities. In L. Subotic (Ed.), English studies today: Views and voices (pp. 175-185). Novi Sad: University of Novi Sad.

Steward, H. (2012). Actions as processes. Philosophical Perspectives, 26(1), 373-388.

Stich, S. (1979). Do Animals have beliefs? Australasian Journal of Philosophy, 57(1), 15-28.

Talmy, L. (1975). Semantics and syntax of motion. In J. P. Kimball (Ed.), Syntax and Semantics 4 (pp. 181-238). New York: Academic Press.

Tardif, T., Gelman, R., \& Xu, F. (1999). Putting the noun bias in context: A comparison of English and Mandarin. Child Development, 70(3), 620-635.

Tranel, D., Manzel, K., Asp, E., \& Kemmerer, D. (2008). Naming dynamic and static actions: Neuropsychological evidence. Journal of Neurophysiology: Paris, 102, 80-94.

Trewavas, A. (2007). Response to Alpi et al.: Plant neurobiology-All metaphors have value. Trends in Plant Science, 12(6), 231-233.

VerbNet (http://verbs.colorado.edu/ mpalmer/projects/verbnet.html).

Wagner, L., \& Carey, S. (2003). Individuation of objects and events: A developmental study. Cognition, 90, $163-191$.

Wilson, M. (2006). Wandering significance: An essay on conceptual behavior. New York: Oxford University Press.

Wimsatt, W. (2006). Reductionism and its heuristics: Making methodological reductionism honest. Synthese, 151, 445-475.

Wynn, K. (1996). Infants' individuation and enumeration of actions. Psychological Science, 7(3), 164-169. 